J. Clin. Chem. Clin. Biochem.

Vol. 17, 1979, pp. 747-755 \title{
Diagnostische Aussagekraft der Kreatinkinase-MB: Verlaufsuntersuchungen bei cardialen und cere-
bralen Erkrankungen
}

Von P. Haux, F. Scheidt ${ }^{1}$ ), Irene Jakob ${ }^{1}$ ), D. Sobe und R. Kattermann

Aus dem Klinisch-Chemischen Institut, Klinikum Mannheim der Universität Heidelberg

(Eingegangen am 12. Juni/1. September 1979)

Dem Gedenken an Prof. Dr. Gábor Szász gewidmet

Zusammenfassung: Der immunologische Inhibitionstest zur Bestimmung der Kreatinkinase-MB erfaßt außer dieser auch das Isoenzym BB, wodurch Fehlinterpretationen verursacht werden können. Bei 239 Patienten mit cardialen und cerebralen Erkrankungen wurde der Wert dieses Tests für die Diagnostik des Herzinfarkts und eine mögliche Störung durch Kreatinkinase-BB untersucht.

Bei 114 Patienten mit cardialen Erkrankungen wurde der Aktivitätsverlauf der Kreatinkinase-MB im Vergleich mit der Gesamt-Kreatin-Kinase, der Aspartat-Aminotransferase und der Hydroxybutyratdehydrogenase im Serum in einem standardisierten Zeitraster gemessen. Während die genannten Enzyme bei 65 Fällen klinisch gesicherter Infarkte einen deutlichen Aktivitätsanstieg im Serum aufwiesen, war bei 3 Patienten (4,5\%) im gesamten Krankheitsverlauf keine Aktivität der Kreatinkinase-MB nachweisbar. Bei 11 Fällen mit Reinfarkt lag der Anteil falsch negativer Befunde sogar nọch höher: Dagegen ergaben sich bei 38 Patienten mit Stenocardie keine falsch positiven Befunde. Für den Nachweis eines Herzinfarktes war 24 Stunden nach Klinikaufnahme der Kreatinkinase-Aspartat-Aminotransferase-Quotient nach $S z a ́ s z$ mit $83 \%$ zuverlässiger als der Quotient Kreatinkinase-MB/Gesamt-Kreatinkinase mit einer Trefferquote von nur $58 \%$.

Bei 125 Patienten mit cerebralen Erkrankungen fanden sich mit unterschiedlicher Häufigkeit Serumaktivitäten der Kreatinkinase-MB über dem sogenannten "Trennwert" von $10 \mathrm{U} / 1$ bzw. über einem Anteil von 0,060 an der Gesamtaktivität. In einzelnen Fällen beobachteten wir paradoxe Quotienten von über 1,00, die den Übertritt von Kreatinkinase-BB aus dem Gehirn wahrscheinlich machten. Mehr als die Hälfte der Patienten mit Schädel-Hirn-Trauma hatte bei der Klinikaufnahme einen Anteil der Kreatinkinase-MB von über 0,060 und zugleich einen Kreatinkinase/AspartatAminotransferase-Quotienten von unter 9. In solchen Fällen kann die Diagnose begleitender oder perioperativ auftretender Myocardschäden oder deren Ausschluß nur durch mehrmalige Untersuchung des Enzymmusters gestützt werden.

Diagnòstic value of creatine kinase-MB: Monitoring in cardiovascular and cerebral diseases

Summary: The immunoinhibition test for the determination of creatine kinase-MB measures the activity of both isoenzymes MB and BB. The presence of creatine kinase-BB therefore leads to misinterpretation. In 239 patients with cardiovascular and cerebral disease we investigated the diagnostic value of this test for myocardial infarction and also the possible interference by creatine kinase-BB.

In 114 patients with cardiovascular disease we measured the serum activities of creatine kinase-MB, total creatine kinase, aspartate aminotransferase and hydroxybutyrate dehydrogenase at standardized blood sampling intervals. In 65 cases of clinically established myocardial infarction we observed a significant increase in activity for the above mentioned enzymes in all but three patients $(4,5 \%)$, whose sera showed no evidence of creatine kinase-MB activity during the whole course of the disease. The percentage of falsely negative cases among 11 patients with myocardial reinfarction was even higher. However, there were no falsely positive results in 38 patients suffering from angina pectoris.

1) Wesentliche Teile dieser Arbeit sind in dẹ Dissertationen von F. Scheidt und I. Jakob enthalten. 
24 hours after admission the creatine kinase/aspartate aminotransferase ratio according to Szász proved to be more reliable for the diagnosis of myocardial infarction than the creatine kinase-MB/ total creatine kinase ratio (in $83 \%$ as compared to $58 \%$ of all cases).

With considerably differing frequency we found creatine kinase-MB activities above $10 \mathrm{U} / \mathrm{l}$ and/or creatine-kinase-MB/ total creatine kinase ratios above 0.060 in 125 patients with cerebral disease. Oçcasionally we observed paradoxical creatine kinase-MB/total creatine kinase ratios above 1.00 , probably due to the presence of creatine kinase- $\mathrm{BB}$ from the brain. At admission more than half of the patients with craniocerebral traumata had a creatine kinase-MB/total creatine kinase ratio over 0.060 and at the same time a creatine kinase/aspartate-aminotransferase ratio below 9. In such cases the diagnosis of concurrent or perioperative myocardial lesions may only be supported by repeated evaluation of the serum enzyme pattern.

\section{Einführung}

Für die Diagnose und Verlaufsbeurteilung des Herzinfarkts werden neben dem EKG als objektive Kriterien die Aktivitätsanstiege einiger myokardialer Enzyme im Serum zugrundegelegt. In der Klinik haben sich für diesen Einsatzbereich die Enzyme Lactatdehydrogenase (EC 1.1.1.27), Aspartat-Aminotransferase (EC 2.6.1.1) und die Kreatinkinase (EC. 2.7.3.2) bewährt. Wie Szász und Mitarbeiter $(1,2)$ erstmals zeigen konnten, erlaubt die Bildung des Quotienten aus Kreatinkinase-und Aspartat-Aminotransferase-Aktivität eine zuverlässige Differenzierung zwischen Erkrankungen des Herzmuskels (Quotient $<9,0$ ) und denen des Skelettmuskels (Quotient $>9,0$ ). Die Berechnung ist jedoch nur zulässig, wenn Erkrankungen der Leber ausgeschlossen sind und die Kreatinkinase $100 \mathrm{U} / 1$ überschreitet. Letzteres ist in der Frühphase des Herzinfarktes häufig nicht der Fall.

Um auch solche Patienten sicher erfassen zu können und generell eine erhöhte diagnostische Aussagekraft bei der Enzymdiagnostik des Herzinfarkts zu erzielen, richtete sich das Interesse der letzten Jahre auf das herzmuskelspezifische Isoenzym der Kreatinkinase, die "Kreatinkinase-MB". Dieses stellt ein Hybrid aus den beiden anderen Isoenzymen der Kreatinkinase dar, der „Kreatinkinase-MM“ (Skelettmuskeltyp) und der „KreatinkinaseBB" (Gehirntyp). Die Bestimmung dieser Isoenzyme durch elektrophoretische bzw. säulenchromatographische Verfahren $(3,4,5)$ war für die Praxis bzw. die Notfalldiagnostik bisher zu unempfindlich und zu zeitaufwendig. Die Entwicklung eines Inhibitionstestes auf immunologischer Basis durch Würzburg et al. bzw. Neumeier et al. $(6,7)$ erlaubt seit wenigen Jahren die rasche und zuverlässige Bestimmung der Kreatinkinase-MB-Aktivität im Serum.

Die ursprünglich für die Kreatinkinase-MB angenommene Herzmuskel- bzw. Infarkt-Spezifität mußte in umfangreichen klinischen Erprobungen allerdings in Zweifel gezogen werden. Dies liegt einmal daran, daß die Kreatinkinase-MB auch in beträchtlicher Aktivität im Skelettmuskel vorkommt (8). Andererseits ist eine gewisse Unsicherheit im Testsystem selbst begründet, das nicht zwischen Kreatinkinase-MB und Kreatinkinase-BB zu unterscheiden vermag. Als Quelle für die KreatinkinaseBB kommt vor allem das Gehirn, und in geringerem Maße die glatte Muskulatur des Magen-Darmtrakts in Frage $(9,10)$

In den hier vorgelegten Ergebnissen untersuch ten wir zunächst an einer größeren $Z$ ahl von Patienten mit cardialen Erkiankungen die diagnostische Zuverlässigkeit des Kreatinkinase/Aspartat-AminotransferaseQuotienten nach Szász $(1,2)$ sowie der K̇reatinkinaseMB bzw. des Kreatinkinase-MB/Gesamt-KreatinkinaseQuotienten. Sodann gingen wir bei Patienten mit cerebralen Erkrankungen der Frage nach, ob und in welchem Umfang mit dem Auftreten von Kreatinkinase bzw. Kreatinkinase-Isoenzymen im Serum zu rechnen ist. Die Ergebnisse dieser in den Jahren 1976-78 durchgeführten Untersuchungen werden in der vorliegenden Arbeit erstmals zusammenfassend dargestellt und diskutiert ${ }^{2}$ ).

\section{Patienten und Methoden}

\section{Patienten}

Nach Eliminierung lückenhafter Verläufe oder nicht bestätigter Verdachtsdiagnosen verblieben zur Auswertung die Daten von 239 Patienten mit cardialen bz̄w. cerebralen Erkrankungen. Die Patienten beider Kollektive wurden in jeweils 3 Gruppen eingeteilt (Tab. 1). Bei den Patienten mit cardialen Erkrankkungen dienten zur Sicherung der Diagnose Anamnese, klinischer Befund und Verlauf, EKG, sowie in einigen Fällen autoptische Untersuchungen. Bei den Patienten mit cerebralen Erkrankungen wirden neben Anamnese und klinischem Befund neurologische und Liquoruntersuchungen, EEG, Carotisangiographie und Computertomographie herangezogen.

Tab. 1. Gruppierung des Krankengutes nach der Hauptsymptomatik.

$$
\text { A. Cardiale Erkrankungen }(n=114)
$$

Diagnose Herzinfarkt Reinfarkt Stenocardie

$\begin{array}{llll}\text { Anzahl } & 65 & 11 & 38\end{array}$

\section{B. Cerebrale Erkrankungen ( $n=125)$}

\begin{tabular}{llll} 
Diagnose & $\begin{array}{l}\text { Hirntumor } \\
\text { Hämatom }\end{array}$ & $\begin{array}{l}\text { Apoplektischer } \\
\text { Insult }\end{array}$ & $\begin{array}{l}\text { Schädel-Hirn- } \\
\text { Trauma }\end{array}$ \\
Anzahl & 37 & 49 & 39 \\
\hline
\end{tabular}

2) Über einen Teil der Ergebnisse wurde bereits auf der gemeinsamen Jahrestagung der Deutschen und Niederländischen Ges. f. Klin. Chemie in Aàchen berichtet (11). 


\section{Methoden}

\section{Probennahme}

In den meisten Fällen wurde Blut ohne Zusätze aus der Cubitalkene gewonnen. Lediglich bei Patienten, die über die Notaufnahme eingewiesen waren, erfolgte die Analytik des Aufnahmebefundes aus organisatorischen Gründen aus Heparinplasma.

Die Blutabnahmen bei den carcialen Erkrankungen wurden in einem fixierten Zeitraster vorgenommen und zwar bei der Aufnahme in die Klinik, nach 12, 24 und 48 Stunden, sowie am 7. Tag des stationären Aufenthaltes. Dieses Schema ließ sich aus verschiedenen Gründen nicht analog auf die cerebralen Erkrankungen übertragen. Es wurden daher nur die Ergebnisse der folgenden Blutabnahmen ausgewertet:

a) bei der Aufnahme in die Klinik bzw. am Tag vor der Operation,

b) am 2. stationären Tag, bzw. am 1. postoperativen Tag (1-7 Stun den nach beendeter Operation),

c) am 3. stationären Tag, bzw. am 2. Tag nach der Operation, und im Falle der operierten Hämatome zusätzlich am 3. postoperativen Tag.

Dic Proben wurden innerhalb 1 Stunde nach Abnahme zentrifugiert und die Seren zur Stabilisierung der Kreatinkinase mit 2-Mercaptoethanol in einer Endkonzentration von $10 \mathrm{mmol} / \mathrm{l}$ versetzt. Bis zur Bestimmung der Enzyme, die spätestens 24 Stunden nach der Blutabnahme erfolgte, wurden die Seren bei $4^{\circ} \mathrm{C}$ aufbewahrt, danach für allfällige Nachuntersuchungen bei $-30^{\circ} \mathrm{C}$ eingefroren.

\section{Best immung der Kreatinkinase-MB}

Die katalytische Konzentration von Kreatinkinase-MB im Serum wurde mit dem MERCK-Test (Art. Nr. 14300) bestimmt, der einen spezifischen, die Untereinheit Kreatinkinase-M vollständig inhibierenden Antikörper enthält (6). Die nicht gehemmte Aktivität der Kreatinkinase-Untereinheit B läßt sich im gekoppelten, enzymatisch-optischen Test in gleicher Weise wie die GesamtKreatinkinase kinetisch messen.

Zur Steigerung der geringen Eimpfindlichkeit des Tests verdoppelten wir den relativen Serumanteil durch Zugabe von $50 \mu \mathrm{l}$ Serum zu $500 \mu \mathrm{l}$ Reaktionsgemisch. Hierdurch ergaben sich geringfügig erniedrigte Endkonzentrationen im Test: Triethanolamin-Puffer $95 \mathrm{mmol} / 1, \mathrm{pH} 7,0$, Kreatinphosphat $33 \mathrm{mmol} / \mathrm{l}$, Glucose $19 \mathrm{mmol} / 1$, Glutathion $8,5 \mathrm{mmol} / 1$, Magnesiumacetat $9,5 \mathrm{mmol} / 1$, ADP $0,95 \mathrm{mmol} / 1$, NADP $0,57 \mathrm{mmol} / 1$, AMP 9,5 mmol/1; Hexokinase 1,2 kU/1, Glucose-6-phosphat-Dehydrogenase 1,2 kU/1, Anti-Kreatinkinase-MM (Ziege), $800 \mathrm{U} / 1$ inhibierend.

Die Absorptionszunahme wurde am Photometer Eppendorf mit Schreiber verfolgt (Schichtdicke $1 \mathrm{~cm}$, Wellenlänge $334 \mathrm{~nm}$, Meßtemperatur $25^{\circ} \mathrm{C}$, Papiervorschub $0,5 \mathrm{~cm} / \mathrm{min}$, Skalenspreizung 0-1,0). In dieser Ausführung resultiert eine etwa viermal größere Empfindlichkeit gegenüber der Originalmethode. Bei der Winkelauswertung des Absorptionsanstiegs im linearen Teil wird die Kreatinkinase-MB-Aktivität nach der Formel:

$$
\mathrm{U} / \mathrm{l}=\tan \alpha \times 89
$$

erriechnet.

Die Vorinkubationszeit und das Meßintervall betrugen jeweils 10 statt 7 Minuten. Seren mit hoher Gesamt-KreatinkinaseAktivität wurden zuvor auf Werte $<500 \mathrm{U} / \mathrm{l}$ verdünnt, obwohl die Inhibitionskapazität des Antikörpers auch im abgewandelten Ansatz mindestens $800 \mathrm{U} / 1$ betrug.

Für die unspezifische Absorptionszunahme registrierten wir im

Mittel eị Áquivalent von $2,1 \mathrm{U} / 1$ mit einer Standardabweichung von $\pm 0,9 \mathrm{U} / 1$. Der Leerwert lag häufig unter $1 \mathrm{U} / 1$, stieg aber gelegentlich bis $4 \mathrm{U} / 1$ an, wobei eine gewisse Chargenabhängigkeit $\mathrm{zu}$ bestehen schien. Auch Würzburg et al. (12) und Klapdor et al. (13) berichten über Leerwerte ähnlicher Höhe. Bei der Berechnung der Kreatinkinase-MB-Aktivität in Serumproben wurde der Leerwert jedoch vernachlässigt. Bei einigen Patientenseren beobachteten wir im Testansatz im Gegensatż zum Leerwert eine geringfuigige Absorptionsabnahme und damit eine scheinbar negative Kreatinkinase-MB-Aktivität. Sie dürfte am ehesten mit einer Konkurrenzreaktion der Glutathionreduktase zu erklären sein (14), obwohl Seren mit sichtbarer Hämolyse nicht zur Mes- sung gelangten. Es ist aber auch eine Abhängigkeit dieses Phänomens vom Eiweißgehalt der Seren postuliert worden (15). Die Absolutwerte dieser scheinbar negativen Aktivitäten betrugen 0,8-3,0 U/1. Für die Tabellen und zur Berechnung der Mittelwerte wurde solchen Seren eine Kreatinkinase-MB-Aktivität von null $\mathrm{U} / \mathrm{l}$ zugeordnet.

\section{Zuverlässigkeitskriterien}

Der gesamte, enzymatische und immunologische Reaktionsablauf ließ sich mit Kreatinkinase-MB-haltigen Humanseren unterschiedlichen Gehalts überprüfen. Es ergab sich auch im veränderten Testansatz nach Inhibition Linearität der Reaktion über $30 \mathrm{~min}$ bis zu einer katalytischen Konzentration von mindestens $123 \mathrm{U} / \mathrm{l}$ (s. Abb. 1). Der erhöhte Serumanteil am Testansatz macht eine Kontrolle der enzymatischen Reaktion erforderlich. Durch die Verwendung eines Kontrollserums auf Albuminbasis mit hochgereinigter Kreatinkinase aus Kaninchenmuskel (Precinorm E der Fa. Boehringer Mannheim) ließ sich der enzymatische von dem immunologischen Reaktionsschritt isolieren. Die erhaltenen Werte für die Kurz- und Langzeitpräzision sind in der Tabelle 2 aufgeführt.

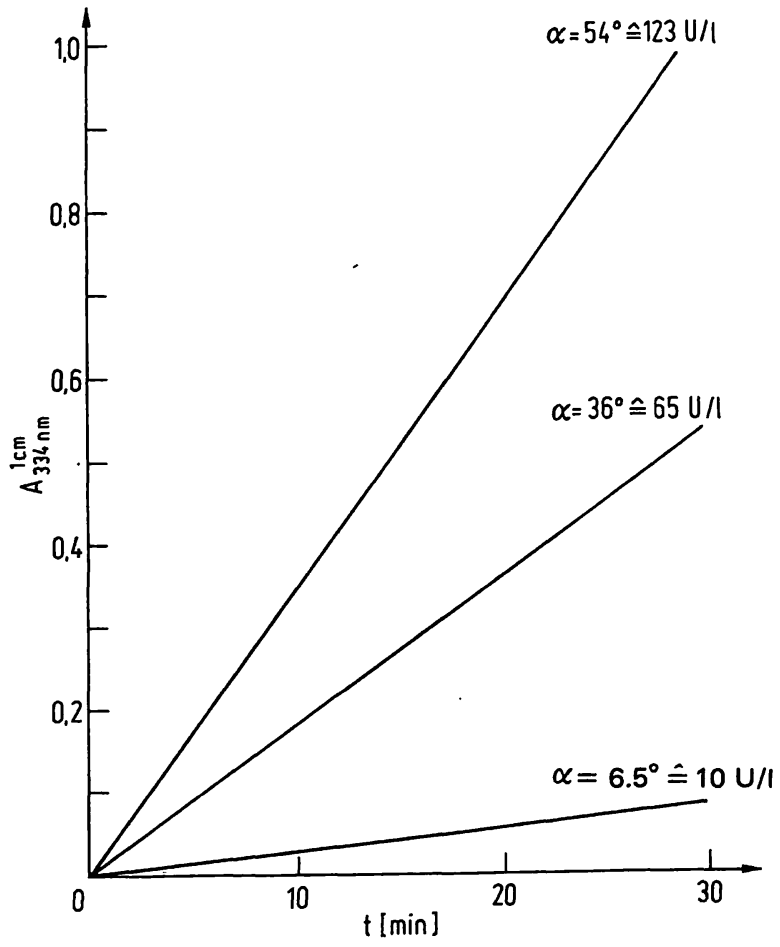

Abb. 1. Linearität der Kreatinkinase-MB-Aktivität im veränderten Testansatz mit Humanseren mit Kreatinkinase-MB-Aktivitäten von 10 sowie 65 und $123 \mathrm{U} / \mathrm{l}$.

Papiervorschub $5 \mathrm{~mm} / \mathrm{min}$.

Tab. 2. Variationskoeffizienten für die Kurz- und Langzeitpräzision der modifizierten Kreatinkinase-MB-Bestimmung.

\begin{tabular}{lllll}
\hline Kontrollserum & \multicolumn{4}{l}{ Precinorm E (1:10) } \\
& $\begin{array}{l}\overline{\mathrm{x}} \\
(\mathrm{U} / \mathrm{l})\end{array}$ & $\begin{array}{l}\text { VK } \\
(\%)\end{array}$ & $\begin{array}{l}\overline{\mathrm{x}} \\
(\mathrm{U} / \mathrm{l})\end{array}$ & $\begin{array}{l}\text { VK } \\
(\%)\end{array}$ \\
\hline $\begin{array}{l}\text { Kurzzeitpräzision } \\
(\mathrm{n}=20)\end{array}$ & 20 & 3,4 & 60 & 1,5 \\
$\begin{array}{l}\text { Langzeitpräzision } \\
(\mathrm{n}=17)\end{array}$ & 22 & 6,9 & 66 & 4,4 \\
\hline
\end{tabular}


Elektrophoretische Trennung der Kreatinkinase-Isoenzyme In einer Reihe von Fällen wurde eine elektrophoretische Iso: enzymtrennung in dünnen Agarose-Gelen durchgeführt (4). Bei Seren mit niedrigen Enzymaktivitäten wurde die Nachweisempfindlichkeit durch den Einsatz dicker Agarose-Gele mit entsprechend höherer Kapazität gesteigert. Die zum Sichtbarmachen der Isoenzymbanden benötigten Bioreagenzien (3) sind als Testkombination käuflich (Sigma Chemical Company, St. Louis, Missouri, Art. Nr. 745-A).

\section{Weitere Enzymbestimmungen}

Gesamt-Kreatinkinase, Aspartat-Aminotransferase und $\alpha$-Hydroxybutyratdehydrogenase wurden im Serum mit optimierten Standardmethoden entsprechend den Empfehlungen der Deutschen Gesellschaft für Klinische Chemie bestimmt (16).

\section{Ergebnisse und Diskussion}

\section{Patienten mit cardialen Erkrankungen}

Das Kollektiv gliedert sich in Patienten mit Herzinfarkten, Reinfarkten und Stenocardien. Die in allen drei Gruppen gemessenen mittleren kataly tischen Konzentrationen und deren Extremwerte sind in Tabelle 3 so aufgeführt, daß die zu gleichen Blutabnahmezeiten gewonnenen Werte untereinander stehen.

\section{Herzinfarkte $(n=65)$}

Die Patienten dieser Gruppe zeigten schon im Aufnahmebefund eine durchschnittliche Kreatinkinase-MB von $11 \mathrm{U} / \mathrm{l}$. Nach 12 und 24 Stunden waren im Mittel jeweils 39 U/l erreicht. Nach 48 Stunden fiel die Kreatinkinase-MB stark ab und war am 7. Tag nur noch in Ausnahmefällen nachweisbar. Die mittlere Aktivität der Gesamt-Kreatinkinase erreichte nach 24 Stunden ein Maximum, die der Kreatinkinase-MB zwischen 12 und 24 Stunden. Bei 70,2\% der Patienten fielen die gemessenen Höchstwerte der Kreatinkinase-MB und der Gesamt-Kreatinkinase zeitlich zusammen, nur in 17,5\% der Fälle wurde die höchste Kreatinkinase-MBAktivität vor und in $12,3 \%$ sogar nach der höchsten Gesamtaktivität erreicht.

Mit dem Nachweis von Kreatinkinase-MB in der Skelettmuskulatur (8) gewann die Berechnung des KreatinkinaseMB-Anteils an der Gesamt-Aktivität an differentialdiagnostischer Bedeutung, weil erst Werte über 0,060 die Skelettmuskulatur als Kreatinkinase-MB-Quelle unwahrscheinlich machen. Bei unserem Krankengut war der höchste durchschnittliche Kreatinkinase-MB-Anteil mit 0,081

Tab. 3. Patienten mit cardialen Erkrankungen $(n=114)$.

Mittel- und Extremwerte der Kreatinkinase-MB (CK-MB), Gesamt-Kreatinkinase (CK gesamt $_{\text {) }}$, Aspartat-Aminotransferase (GOT) und $\alpha$-Hydroxybutyrat-Dehydrogenase $(\alpha-\mathrm{HBDH})$ zu verschiedenen Blutentnahmezeiten.

\begin{tabular}{|c|c|c|c|c|c|}
\hline Zeitpunkt & Aufnahme & $12 \mathrm{~h}$ & $24 \mathrm{~h}$ & $48 \mathrm{~h}$ & 7. Tag \\
\hline & \multicolumn{5}{|c|}{ Herzinfarkte $(n=65)$} \\
\hline CK-MB (U/l) & $\begin{array}{l}11 \\
(0-130)\end{array}$ & $\begin{array}{l}39 \\
(0-186)\end{array}$ & $\begin{array}{l}39 \\
(0-175)\end{array}$ & $\begin{array}{l}10 \\
(0-48)\end{array}$ & $\begin{array}{c}1 \\
(0-6)\end{array}$ \\
\hline $\mathrm{CK}_{\text {gesamt }}(\mathrm{U} / \mathrm{l})$ & $\begin{array}{l}155 \\
(12-1100)\end{array}$ & $\begin{array}{l}463 \\
(33-2563)\end{array}$ & $\begin{array}{l}543 \\
(33-1450)\end{array}$ & $\begin{array}{l}213 \\
(21-750)\end{array}$ & $\begin{array}{l}27 \\
(6-161)\end{array}$ \\
\hline GOT (U/l) & $\begin{array}{l}41 \\
(10-217)\end{array}$ & $\begin{array}{l}95 \\
(11-368)\end{array}$ & $\begin{array}{l}127 \\
(14-1104)\end{array}$ & $\begin{array}{l}63 \\
(10-187)\end{array}$ & $\begin{array}{l}39 \\
(8-910)\end{array}$ \\
\hline \multirow[t]{2}{*}{$\alpha-\mathrm{HBDH}(\mathrm{U} / \mathrm{l})$} & $\begin{array}{l}235 \\
(94-915)\end{array}$ & $\begin{array}{l}419 \\
(108-1145)\end{array}$ & $\begin{array}{l}634 \\
(101-2145)\end{array}$ & $\begin{array}{l}640 \\
(110-2145)\end{array}$ & $\begin{array}{l}297 \\
(97-1012)\end{array}$ \\
\hline & \multicolumn{5}{|c|}{ Reinfarkte ( $n=11)$} \\
\hline CK-MB (U/1) & $\begin{array}{c}5 \\
(0-17)\end{array}$ & $\begin{array}{l}12 \\
(0-63)\end{array}$ & $\begin{array}{l}13 \\
(0-80)\end{array}$ & $\begin{array}{c}5 \\
(0-13)\end{array}$ & $\begin{array}{c}1 \\
(0-6)\end{array}$ \\
\hline $\mathrm{CK}_{\text {gesamt }}(\mathrm{U} / \mathrm{l})$ & $\begin{array}{l}69 \\
(17-1346)\end{array}$ & $\begin{array}{l}160 \\
(46-503)\end{array}$ & $\begin{array}{l}311 \\
(30-1503)\end{array}$ & $\begin{array}{l}162 \\
(7-945)\end{array}$ & $\begin{array}{l}15 \\
(9-128)\end{array}$ \\
\hline GOT (U/l) & $\begin{array}{l}45 \\
(9-158)\end{array}$ & $\begin{array}{l}70 \\
(13-263)\end{array}$ & $\begin{array}{l}136 \\
(12-748)\end{array}$ & $\begin{array}{l}54 \\
(9-228)\end{array}$ & $\begin{array}{l}17 \\
(10-50)\end{array}$ \\
\hline \multirow[t]{2}{*}{$\alpha-\mathrm{HBDH}(\mathrm{U} / \mathrm{l})$} & $\begin{array}{l}241 \\
(116-988)\end{array}$ & $\begin{array}{l}350 \\
(145-1060)\end{array}$ & $\begin{array}{l}431 \\
(150-1430)\end{array}$ & $\begin{array}{l}324 \\
(119-1065)\end{array}$ & $\begin{array}{l}211 \\
(136-572)\end{array}$ \\
\hline & \multicolumn{5}{|c|}{ Stenocardien $(n=38)$} \\
\hline CK-MB (U/l) & $\begin{array}{c}1 \\
(0-6)\end{array}$ & $\begin{array}{c}2 \\
(0-6)\end{array}$ & $\begin{array}{c}1 \\
(0-6)\end{array}$ & $\begin{array}{c}1 \\
(0-3)\end{array}$ & $\begin{array}{c}1 \\
(0-4)\end{array}$ \\
\hline $\mathrm{CK}_{\text {gesamt (U/l) }}$ & $\begin{array}{l}20 \\
(4-65)\end{array}$ & $\begin{array}{l}29 \\
(7-107)\end{array}$ & $\begin{array}{l}33 \\
(7-150)\end{array}$ & $\begin{array}{l}22 \\
(4-100)\end{array}$ & $\begin{array}{l}14 \\
(3-41)\end{array}$ \\
\hline GOT (U/l) & $\begin{array}{l}16 \\
(9-44)\end{array}$ & $\begin{array}{l}18 \\
(6-61)\end{array}$ & $\begin{array}{l}15 \\
(6-47)\end{array}$ & $\begin{array}{l}14 \\
(7-30)\end{array}$ & $\begin{array}{l}17 . \\
(10-37)\end{array}$ \\
\hline$\alpha-\mathrm{HBDH}(\mathrm{U} / \mathrm{l})$ & $\begin{array}{l}145 \\
(58-261)\end{array}$ & $\begin{array}{l}157 \\
(62-346)\end{array}$ & $\begin{array}{l}150 \\
(84-404)\end{array}$ & $\begin{array}{l}139 \\
(92-275)\end{array}$ & $\begin{array}{l}1.43 \\
(6.7-283)\end{array}$ \\
\hline
\end{tabular}


Tab. 4. Patienten mit cardialen Erkrankungen.

Mittel- und Extremwerte der individuellen Kreatinkinase-MB-Anteile an der Gesamt-Kreatinkinase-Aktivität $\frac{\text { CK-MB }}{\mathrm{CK}_{\text {gesamt }}}$ und der Kreatinkinase/Aspartat-Aminotransferase-Quotienten $\frac{\mathrm{CK}}{\mathrm{GOT}}$ nach Szász bei frischen Infarkten und Reinfarkten.

\begin{tabular}{|c|c|c|c|c|}
\hline Zeitpunkt & Aufnahme & $12 \mathrm{~h}$ & $24 \mathrm{~h}$ & $48 \mathrm{~h}$ \\
\hline & \multicolumn{4}{|c|}{ Herzinfarkte ( $n=63)$} \\
\hline$\frac{C K-M B}{C_{\text {gesamt }}}$ & $\begin{array}{l}0,046 \\
(0-0,462)\end{array}$ & $\begin{array}{l}0,081 \\
(0-0,213)\end{array}$ & $\begin{array}{l}0,076 \\
(0-0,180)\end{array}$ & $\begin{array}{l}0,065 \\
(0-0,335)\end{array}$ \\
\hline$\frac{\mathrm{CK}}{\mathrm{GOT}}$ & $\begin{array}{l}5,1 \\
(1,9-13,5)\end{array}$ & $\begin{array}{l}4,9 \\
(2,5-12,5)\end{array}$ & $\begin{array}{l}5,0 \\
(1,1-16,7)\end{array}$ & $\begin{array}{l}4,3 \\
(1,4-25,0)\end{array}$ \\
\hline & \multicolumn{3}{|c|}{ Reinfarkte $(n=11)$} & \\
\hline$\frac{\mathrm{CK}-\mathrm{MB}}{\mathrm{CK}_{\text {gesamt }}}$ & $\begin{array}{l}0,105 \\
(0-0,278)\end{array}$ & $\begin{array}{l}0,095 \\
(0-0,405)\end{array}$ & $\begin{array}{l}0,084 \\
(0-0,206)\end{array}$ & $\begin{array}{l}0,078 \\
(0-0,308)\end{array}$ \\
\hline$\frac{\mathrm{CK}}{\mathrm{GOT}}$ & $\begin{array}{l}3,5 \\
(2,2-4,8)\end{array}$ & $\begin{array}{l}4,1 \\
(1,3-5,0)\end{array}$ & $\begin{array}{l}4,9 \\
(1,9-8.7)\end{array}$ & $\begin{array}{l}4,5 \\
(0,9-9,6)\end{array}$ \\
\hline
\end{tabular}

nach 12 Stunden erreicht (Tab. 4). Bei der Aufnahme lag nur bei 18 von 64 Patienten (28\%) ein Kreatinkinase-MBAnteil von 0,060 oder mehr vor. Nach 12 Stunden er. füllten 41 Patienten (64\%) dieses Kriterium. Danach fiel die Zahl über $37(24 \mathrm{~h})$ auf 18 Patienten $(48 \mathrm{~h})$ ab.

Der Verlauf der Kreatinkinase-MB-Aktivitäten steht im Einklang mit den Daten von Neumeier et al. (17), die bei häufigerer Probennahme das Maximum der KreatinkinaseMB im Mittel 17,4 Stunden nach Beginn des Thoraxschmerzes beobachteten. Allerdings fanden diese Autoren im Maximum der Gesamtaktivität eine mit 62 U/1 höhere durchschnittliche Kreatinkinase-MB-Aktivität. Letztere betrug bei unserem eigenen Krankengut 39 U/1. Bei einer wenig höheren mittleren Gesamt-Kreatinkinase-Aktivität von $585 \mathrm{U} / 1$ ergibt sich ein mit 0,132 deutlich höherer Kreatinkinase-MB-Anteil. In früheren Mitteilungen der gleichen Autoren $(6,18)$ finden sich dageben ebenfalls Kreatinkinase-MB-Anteile von etwa 0,080. Die beobachteten Differenzen dürften ihre Ursache im gewählten Zeitraster und in einem größeren Anteil schwerer Myocardnekrosen bei Neumeier et al. (17) haben.

Für den Kreatinkinase/Aspartat-AminotransferaseQuotientẹn nach Szász (s. Tab. 4) waren bei der Aufnahme in 23 von 64 Fällen (36\%) die eingangs genannten Voraussetzungen erfüllt. In 22 dieser 23 Fälle $(95,7 \%)$ lag dieser Quotient mit Werten $<9$ in dem für Herzinfarkte typischen Bereich. Nach 12 Stunden lauteten die Żahlen 48 von 50 (96\%), nach 24 Stunden 53 von $55(96,4 \%)$ und nach 48 Stunden 37 von 38 Patienten (97,4\%). Sie demonstrieren die hohe Treffsicherheit des Szász-Quotienten, wenn eịnige Einschränkungen beachtet werden. Aber selbst wenn die Trefferquote des Kreatinkinase/Aspartat-Aminotransferase-Quotienten am Gesamtkollektiv unter Einschluß der nicht für die Berechnung des Quotienten geeigneten Verläufe gemessen wurde, ergab sich für den Szász-Quotienten zu allen Blutabnehmezeiten ein höherer Anteil testpositiver Ergebnisse als für den Kreatinkinase-MB/ Gesamt-Kreatinkinase-Quotienten. Die Trefferquote bei Chemnitz et al. (19) $93,6 \%$ - erreichte mit $83 \%$ nach 24 Stunden ein Maximum, während sie für den Kreatinkinase-MB/Gesamt-Kreatinkinase-Quotienten nur $58 \%$ betrug. Neben dem Kreatinkinase-MB/GesamtKreatinkinase-Quotienten gilt als Entscheidungskriterium eine Kreatinkinase-MB-Aktivität von $>10 \mathrm{U} / 1$. Dieser "Trennwert" muß hauptsächlich wegen der geringen Empfindlichkeit der Originalmethode so hoch angesetzt werden. Dies bedeutet, daß erst bei GesamtKreatinkinase-Aktivitäten ab 160 U/l ein KreatinkinaseMB-Anteil von 0,060 sicher gemessen werden kann. In unserer Modifikation lag die technische Nachweisgrenze, definiert als mittlerer Leerwert $+3 \mathrm{~s}$, bei $4,8 \mathrm{U} / \mathrm{l}$. Sie erlaubte eine Herabsetzung des Trennwerts auf $5 \mathrm{U} / \mathrm{l}$ und damit eine bessere Beurteilung von niedrigen Gesamt-Kreatinkinase-Aktivitäten ab 80 U/1. Mit dem niedrigeren Trennwert betrug der Anteil testpositiver Patienten bei der Klinikaufnahme 36 statt 23\% und erreicht die Trefferquote des Kreatinkinase/AlaninAminotransferase-Quotienten. Bei der zweiten und dritten Blutentnahme ergaben sich nur geringe Unterschiede bei der Verwendung der niedrigeren Trennwerte.

Bei 3 Patienten lieferte die Kreatinkinase-MB-Bestimmung während des gesamten Krankheitsverlaufs keine verwertbaren Aktivitätsanstiege. Nur bei einem Patienten erreichte ein einzelner Wert den Trennwert von $5 \mathrm{U} / \mathrm{l}$. $\hat{\mathrm{K}}$ linik, EKG und infarkttypische Verläufe für die Aktivitäten der Gesamt-Kreatinkinase, Aspartat-Aminotrans- 
ferase und $\alpha$-Hydroxybutyrat-Dehydrogenase stützten die Diagnosen eines Anteroseptal-, Vorderwand- und Hinterwandinfarktes. Dies spricht im Gegensatz zu früheren Mitteilungen $(20,21)$ für das Vorkommen falsch negativer Befunde. Die statistischen Daten einer jüngeren Arbeit von Neumeier et al. (17) ergeben einen Anteil falsch negativer Befunden von mindestens $3 \%$. Flenker et al. (22) berichten sogar über $7 \%$, so daß die bei unserem Krankengut beobachtete $Z$ ahl von $4,5 \%$ falsch negativen Befunden realistisch erscheint.

Für die Kreatinkinase-MB-Bestimmung ergibt sich somit kein statistisch faßbarer Vorteil gegenüber dem Kreatin: kinase/Aspartat-Aminotransferase-Quotienten. Ihr Wert wird allerdings dadurch erhöht, daß bei der Aufnahme 5 von 41 Patienten, deren Kreatinkinase-Aktivitäten im Serum eine Quotientenbildung nach Szász nicht erlaubten. Kreatinkinase-MB-Aktivitäten über 5 U/1 aufwiesen. Beide Verfahren können sich somit ergänzen.

\section{Reinfarkte $(n=11)$}

Bei dieser kleinen Gruppe von Patienten fiel zunächst auf, daß die mittleren Aktivitäten der Kreatinkinase, der Kreatinkinase-MB und der $\alpha$-Hydroxybutyrat-Dehydrogense zu allen Zeitpunkten erheblich unter den entsprechenden Werten des Infarktkollektivs lagen (s. Tab. 3). Zugleich durchliefen alle mittleren Aktivitäten nach 24 Stunden ein Maximum. Der Anteil der Kreatinkinase-MB an der Gesamtaktivität (s. Tab. 4) war mit 0,105 schon bei der ersten Blutabnahme am höchsten. Er lag bei allen frühen Blutabnahmen über den korrespondierenden Werten der Gruppe der Infarkte. Dieser Befund steht qualitativ und quantitativ im Einklang mit Neumeier et al. $(18,7)$. Die Kreatinkinase/Aspartat-AminotransferaseQuotienten (s. Tab. 4) lagen mit Ausnahme einzelner Extremwerte weit unter 9, waren aber wegen der häufig niedrigen Kreatinkinase-Aktivitäten nur in der Hälftè der Fälle anwendbar.

Trotz des im -Vergleich zu den Infarkt-Patienten durchschnittlich höheren Anteils der Kreatinkinase-MB an der Gesamt-Aktivität, wiesen maximal $60 \%$ der Patienten Kreatinkinase-MB-Anteile von 0,060 und darüber auf, und nur bei $70 \%$ der Patienten war die Nachweisgrenze für Kreatinkinase-MB von 4,8 U/l überschritten. Dieses Verhalten spricht für einen relativ hohen Anteil falsch negativer Befunde: Bei 3 von 11 Patienten lag der gefundene Kreatinkinase-MB-Wert in allen Serumproben mit 0-2 U/1 unter dem mittleren Leerwert. Einer dieser Patienten, in dessen Krankheitsverlauf die übrigen Infarktenzyme überraschenderweise beträchtliche.Aktivitäten erreichten (Kreatinkinase 1505, Aspartat-Aminotransferase 748 und $\alpha$-Hydroxybuty rat-Dehydrogenase 1430 U/1 nach 24 Stunden), verstarb am 7. Tag. Der Sektionsbefund bestätigte die Diagnose eines ausgedehnten frischen Infarktrezidivs im Bereich der linksventriculären.Vorderwand. Bei einem weiteren, ebenfalls durch Autopsie gesicherten Fall, ließ sich lediglich ein einziges Mal nach 12 Stunden Kreatinkinase-MB-Aktivität von $9 \mathrm{U} / 1$ entsprechend einem Anteil von 0,025 an der Gesamt-Aktivität nachweisen. Ein durch Glutathionreduktase methodisch bedingter negativer Ausfall des Isoenzymnachweises ist nicht wahrscheinlich. Neuerdings steht ein Testsystem mit $\mathrm{N}$-Acetylcystein zur Verfügung, das diese Störung ausschließt. Interessanterweise teilten Schmidt \& Bender (23) kürzlich in dieser Zeitschrift mit, daß zwischen der alten Glutathionmethode und der N-Acetylcysteinmethode eine sehr enge Korrelation besteht.

\section{Patienten mit Stenocardien ( $n=38)$}

Die Patienten dieser Gruppe wurden wegen starker stenocardischer Beschwerden stationär aufgenommen. Nur in Ausnahmefällen konnten wir geringfügige Erhöhungen der Enzymaktivitäten über die obere Normgrenze beobachten. Die höchste Kreatinkinase-MB-Aktivität betrug $6 \mathrm{U} / 1$; sie wurde bei 169 Einzelmessungen viermal beobachtet $(2,4 \%)$. Insgesamt 10 Werte $(5,9 \%)$ überschritten den Grenzwert von 4,8 U/1. Nur bei einem Patienten lagen die Kreatinkinase-MB-Aktivitäten bei zwei aufeinanderfolgenden Blutabnahmen knapp über dem Trennwert von $5 \mathrm{U} / 1$, wobei der zweite Wert bereits eine fallende Tendenz zeigte. Die Wahrscheinlichkeit, mit der bei diesem Kollektiv ein Infarkt ausgeschlossen werden kann, wird durch eine im Abstand von 6-12 Stunden wiederholte Messung der Kreatinkinase-MBAktivität erheblich gesteigert, vor allem, wenn die Erstbestimmung einen Grenzwērt ergab. Auch Schmidt et al. (24) haben bei ihren Patienten mit Stenocardien keine falsch positiven Ergebnisse erhalten, so daß der Kreatinkinase-MB-Bestimmung gerade hier eine besondere $\mathrm{Be}$ deutung zum Ausschluß eines Infarktes zukommt.

\section{Patienten mit cerebralen Erkrankungen}

Die Patienten mit cerebralen Erkrankungen lasșen sich drei Gruppen zuordnen (Tab. 1). In der 1. Gruppe sind Patienten mit raumfordernden Prozessen des Schädels zusammengefaßt. Ursache der Hirndrucksy mptomatik, die neuro-chirurgische Maßnahmen erzwang, waren Tumoren und - teilweise traumatisch bedingte - Hämatome. Die 2. Gruppe umfaßt Patienten mit apoplektischen Insulten ohne Drucksteigerung im Schädel und die 3. Gruppe Patienten mit Schädel=Hirn-Traumen. Bei letzterer Gruppe handelt es sich überwiegend um Opfer von Unfällen, die zu Frakturen des Schädels und/oder zur Contusio cerebri führten. Häufig lagen zugleich mehr oder weniger schwere Verletzungen der Extremitäten vor.

\section{Hirntumoren und Hämatome ( $n=37)$}

Trotz ähnlicher Symptomatik und Therapie zeigten die gemessenen Enzymaktivitäten bei den Patienten mit Tumoren und Hämatomen so deutliche Unterschiede, daß eine getrennte Auswertung. der Daten geboten war. 
Tab. 5. Patienten mit raumfordernden Prozessen des Schädels.

Mittel- und Extremwerte der Kreatinkinase-MB- (CK-MB) und der Gesamt-Kreatinkinase-Aktivitäten (CK gesamt ).

\begin{tabular}{|c|c|c|c|c|}
\hline Zeitpunkt & präoperativ & 1. Tag post op. & 2. Tag post op. & 3. Tag post op. \\
\hline & \multicolumn{4}{|c|}{ Hämatome ( $n=20)$} \\
\hline $\mathrm{CK}-\mathrm{MB}(\mathrm{U} / \mathrm{l})$ & $\begin{array}{l}13 \\
(0-36)\end{array}$ & $\begin{array}{c}12 \\
(0-77)\end{array}$ & $\begin{array}{c}9 \\
(0-48)\end{array}$ & $\begin{array}{c}9 \\
(0-60)\end{array}$ \\
\hline \multirow[t]{2}{*}{$\mathrm{CK}_{\text {gesamt }}(\mathrm{U} / \mathrm{l})$} & $\begin{array}{l}163 \\
(15-618)\end{array}$ & $\begin{array}{l}182 \\
(9-840)\end{array}$ & $\begin{array}{l}246 \\
(22-1032)\end{array}$ & $\begin{array}{l}248 \\
(11-896)\end{array}$ \\
\hline & \multicolumn{4}{|c|}{ Hìrntumoren $(n=17)$} \\
\hline CK-MB (U/1) & $\begin{array}{c}1 \\
(0-1)\end{array}$ & $\begin{array}{c}2 \\
(0-9)\end{array}$ & $\begin{array}{c}3 \\
(0-9)\end{array}$ & - \\
\hline $\mathrm{CK}_{\text {gesamt }}(\mathrm{U} / \mathrm{l})$ & $\begin{array}{c}10 \\
(3-53)\end{array}$ & $\begin{array}{l}100 \\
(7-217)\end{array}$ & $\begin{array}{l}81 \\
(13-271)\end{array}$ & - \\
\hline
\end{tabular}

Im Serum der Tumorpatienten $(\mathrm{n}=17)$ fanden sich im gesamten Beobachtungszeitraum nur geringe Kreatinkinase-MB-Aktivitäten. Präoperativ lagen sämtliche Meßwerte mit 0-2 U/1 im Bereich des Leerwerts. Bei 34 postoperativen Blutproben wurden $9 \mathrm{mal}$ KreatinkinaseMB-Aktivitäten zwischen 5 und 9 U/l beobachtet. Die durchschnittlichen Kreatinkinase-MB-Aktivitäten erreichten daher auch nach der Operation nur $3 \mathrm{U} / 1$ (Tab. 5)

Der Anteil der Kreatinkinase-MB an der Gesamtaktivität, die am 1. postoperativen Tag mit $100 \mathrm{U} / 1$ den höchsten Mittelwert erreichte, betrug nur bei 6 postoperativen Blutabnahmen mehr als 0,060 mit einem Höchstwert von 0,366 (Tab. 8). Da das Testsystem nicht zwischen Kreatinkinase-MB und Kreatinkinase-BB unterscheidet, könnte es sich bei diesen Patienten um Kreatinkinase-BB handeln. Dies bedeutet eine Einschränkung des Werts der Kreatinkinase-MB-Bestimmung im Rahmen einer perioperativen, kardiologischen Diagnostik. In Zweifelsfällen vermag eine nach einigen Stunden wiederholte Kreatinkinase-MBBestimmung den nötigen Aufschluß zu geben: Bei Myokardschädigung ist mit einem deutlichen Aktivitätsanstieg zu rechnen.

Bei den Patienten mit Hämatomen waren im Gegensatz zum Krankengut mit Tumoren vor und nach dem neurochirurgischen Eingriff erhebliche Kreatinkinase-MBAktivitäten nachweisbar. Der höchste Einzelwert von $77 \mathrm{U} / 1$ wurde am ersten postoperativen Tag gemessen, die höchște mittlere Aktivität vọn $13 \mathrm{U} / 1$ ergab sịch jedoch sçhon präoperativ. Die mittlere Aktivität fiel kontinuierlich auf $9 \mathrm{U} / 1$ am 3. postoperativen Tag ab. (Tab. 5).

Wegen der begleitenden Únfalliverletzungen bei einem Teil der Patienten lagen die durchschnittlichen GesamtKreatinkinase-Aktivitäten erheblich über denen das Tumorkollektivis und stiegen im Gegensatz zu den mittleren Kreatinkinase-MB-Aktivitäten kontinuierlich an. Diese Gegenläufigkeit der Aktivitäten führte zu einem Kreatinkinase-MB/Gesamt Kreatinkinase-Quotienten, der von 0,104 (präoperativ) rasch auf 0,020 am 3. postoperativen Tag fiel (Tab. 8).

Ein besonders auffälliges Ergebnis, bei dem die Kreatinkinase-MB-Aktivität erheblich über der Gesamt-Aktivität (Kreatinkinase-MB 77 U/1, Gesamt-Kreatinkinase 55 U/1) lag, blieb bei der Bildung der Mittelwerte unberücksichtigt. Vor der Operation konnte bei diesen Patienten keine Kreatinkinase-MB nachgewiesen werden. Die Annahme einer Störung des Tests durch Kreatinkinase-BB lag nahe. Auch bei den Patienten mit Hämatomen kann es demnach Schwierigkeiten bei der Interpretation einzelner, erhöhter Kreatinkinase-MB-Aktivitäten geben.

\section{Apoplektischer Insult ( $n=49$ )}

In dieser Gruppe sind Patienten mit Apoplexien ( $n=36)$ und Subarachnoidalblutungen $(n=15)$ zusammengefaßt. Von den Mustern der Enzymaktivitäten her ließ sich keine Notwendigkeit zur getrennten Behandlung dieser beiden Gruppen ableiten. Die mittleren Aktivitäten der Kreatinkinase-MB und der Gesamt-Kreatinkinase lagen durchweg in niedrigen Bereichen, wenn auch in Einzelfällen an allen drei Tagen stark erhöhte Werte gemessen wurden (Tab. 6). Die Zahl der testpositiven Patienten stieg von 5 am 1 . Tag auf 11 am 2. Tag und fiel dann auf 8 am 3. Tag.

Der mittlere Anteil der Kreatinkinase-MB an der Gesamtaktivität lag $<0,040$ wenn man die auffällig hohen

Tab. 6. Patienten mit apoplektischem Insult $(n=49)$ Mittel- und Extremwerte der Kreatinkinase-MB (CK-MB) und Kreatinkinase-Gesamt- ( $\left.\mathrm{CK}_{\text {gesamt }}\right)$ Aktivitäten.

\begin{tabular}{lllll}
\hline Zeitpunkt & 1. Tag & & 2. Tag & 3. Tag \\
\cline { 2 - 5 } CK-MB (U/1) & 3 & 4 & 3 \\
& $(0-48)$ & & $(0-38)$ & $(0-42)$ \\
& & & 93 & 92 \\
CK $_{\text {gesamt }}(\mathrm{U} / \mathrm{l})$ & 61 & $(4-876)$ & $(6-710)$ & $(6-878)$ \\
\hline
\end{tabular}


Kreatinkinase-MB-Anteile von 3 Patienten mit Apoplexien nicht in die Berechnung einbezog. Bei einem Patienten lag dieser an allen 3 Tagen über $1,00(1,03-1,26)$, beim zweiten stieg er von 0,45 über 0,61 auf 0,92 und beim dritten Patienten bewegte er sich zwischen 0,19 und 0,40 . Da bei 2 Patienten das normale EKG eine Myocardbeteiligung ausschloß, mußte ein Durchbruch der Blut-Hirn-Schranke mit Übertritt von Kreatinkinase$\mathrm{BB}$ ins strömende Blut angenommen werden. In diesen Fällen lagen die Kreatinkinase-MB-Aktivitäten mit 12$48 \mathrm{U} / \mathrm{l}$ in einem herzinfarkttypischen Bereich, so daß sich Schwierigkeiten in deren Beurteilung ergeben können.

Bei einer Reihe von Patienten mit Hämatomen und Apoplexien schien Kreatinkinase-BB vorzuliegen, deren Aktivität im verwendeten Testsystem um den Faktor 2 überschätzt wird. Auf diese Weise können paradoxe Kreatinkinase-MB-Aktivitäten beobachtet werden, die höher sind als die Gesamt-Kreatinkinase-Aktivität. Befunde dieser Art führten zum Nachweis des seltenen idiopathischen Vorkommens des Isoenzyms KreatinkinaseBB (25). Der direkte Nachweis von Kreatinkinase-BB durch elektrophoretische Trennung der Isoenzyme gelang jedoch nicht. Da tiefgefrorene Seren verwendet wurden, mag die Ursache in einer Instabilität der Kreatinkinase-B-haltigen Isoenzyme liegen, denn auch tiefgefrorene Seren von Infarktpatienten erzeugten nur äußerst schwache Kreatinkinase-MB-Banden, die in ihrer Stärke denen von mitgeführten frischen Seren ähnlichen Kreatinkinase-MB-Gehalts auch nicht annähernd entsprachen. Gerhardt \& Waldenström (26) beobachteten bei chromatographisch isolierter HumanKreatinkinase-MB schon bei einer eintägigen Lagerung bei $4^{\circ} \mathrm{C}$ und nach Einfrieren und Auftauen in ImidazolAcetat-Puffer Dissoziation in die Isoenzyme MM und BB. Über eine ausgeprägte Instabilität von Kaninchen-Kreatinkinase-BB berichten Frotscher et al. (27). Daß sich schon durch kurzfristige Lagerung elektrophoretische Isoenzymmuster durch nichtenzymatische Desamidierung ändern können, hat Lorentz (28) für die Amylasen nachgewiesen.

\section{Schädel-Hirn-Trauma ( $n=39)$}

Bei diesem Kollektiv mit zum Teil schweren Unfallverletzungen fanden sich die höchsten Kreatinkinase-MBAktivitäten im gesamten Krankengut mit cerebralen Erkrankungen. Sie erreichten $127 \mathrm{U} / \mathrm{l}$ am Aufnahmetag bei einer mittleren Aktivität von $28 \mathrm{U} / \mathrm{l}$. Letztere fiel am 2. und 3. Tag auf $18 \mathrm{bzw} .17 \mathrm{U} / \mathrm{l} \mathrm{ab}$, jedoch wurden auch dann noch Extremwerte um $100 \mathrm{U} / 1$ gemessen (Tab. 7). Auffällig war ferner die hohe Zahl testpositiver Patienten, die bei der Klinikaufnahme auf der Basis eines Trennwerts von $5 \mathrm{U} / 187,2 \%$ betrug. Am 2. und 3. Tag waren es $58,6 \%$ und $48,4 \%$. Bei einem Trennwert von $10 \mathrm{U} / \mathrm{l}$ lauteten die entsprechenden Zahlen 69,2\%, 36,8\% und 35,5\%. Im Gegensatz zur Aktivität der Kreatinkinase-MB stieg die der Gesamt-Kreatinkinase vom 1. zum 2. Tag hin steil an. Die Gegenläufigkeit beider Aktivitäten
Tab. 7. Patienten mit Schädel-Hirn-Traumen $(n=39)$ Mittel- und Extremwerte der Kreatinkinase-MB- (CKMB) und Gesamt-Kreatinkinase-( CK $\left._{\text {gesamt }}\right)$ Aktivitäten.

\begin{tabular}{llll}
\hline Zeitpunkt & $1 . \mathrm{Tag}$ & $2 . \mathrm{Tag}$ & $3 . \mathrm{Tag}$ \\
\hline CK-MB (U/1) & 28 & 18 & 17 \\
& $(0-127)$ & $(0-102)$ & $(0-96)$ \\
CK & & 587 & 566 \\
& 252 & $(17-2872)$ & $(17-2154)$ \\
\hline
\end{tabular}

bedingte ähnlich wie bei den Patienten mit intracraniellen Hämatomen einen starken Rückgang des mit 0,172 zuerst hohen Anteils der Kreatinkinase-MB an der Gesamtaktivität auf Werte um 0,030 (Tab. 8). Hackl et al. (29), die darauf hinweisen, daß bei polytraumatisierten Patienten Herzkontusionen auch ohne Rippen- oder Ster: numfrakturen häufig vorkommen, schlagen als Entscheidungshilfe für die Diagnose cardialer Begleit- oder Folgeschäden einen Kreatinkinase-MB-Anteil an der Gesamtaktivität von mindestens 0,100 vor. Bei über der Hälfte der Patienten mit Schädel-Hirn-Traumen war auch dieser Wert am Tag der Aufnahme überschritten, am 2. Tag hingegen nur noch bei 2 von 38 Patienten. Bei der Aufnahme lag außerdem bei der Hälfte der Patienten der Kreatinkinase/Aspartat-Aminotransferase-Quotient zugleich unter 9. Dieses Verhalten zeigt deutlich, daß cardiale Komplikationen nicht aus dem Enzymmuster einzelner Blutabnahmen abgeleitet werden können.

Tab. 8. Patienten mit cerebralen Erkrankungen

Anteil der Kreatinkinase-MB (CK-MB) an der GesamtAktivität ( $\left.\mathrm{CK}_{\text {gesamt }}\right)$. Mittel- und Extremwerte.

\section{A. Raumfordernde Prozesse}

\begin{tabular}{lllll}
\hline Zeitpunkt & $\begin{array}{l}\text { prä- } \\
\text { operativ }\end{array}$ & $\begin{array}{l}\text { 1. Tag } \\
\text { post op. }\end{array}$ & $\begin{array}{l}\text { 2. Tag } \\
\text { post op. }\end{array}$ & $\begin{array}{l}\text { 3. Tag } \\
\text { post op. }\end{array}$ \\
\hline
\end{tabular}

Hämatome $(n=20)$

$\begin{array}{lllll}\mathrm{CK}-\mathrm{MB} & 0,104 & 0,046 & 0,038 & 0,020 \\ \mathrm{CK}_{\text {gesamt }} & \begin{array}{l}(0-0,50) \\ (0-1,40)^{*}\end{array} & (0-0,136) & (0-0,067)\end{array}$

Hirntumoren $(n=17)$

$\begin{array}{llll}\mathrm{CK}-\mathrm{MB} & 0 & 0,035 & 0,024 \\ \mathrm{CK}_{\text {gesamt }} & 0 & (0-0,366) & (0-0,176)\end{array}$

B. Apoplexien und Schädel-Hirn-Traumen

\begin{tabular}{|c|c|c|c|}
\hline Zeitpunkt & 1. Tag & 2. Tag & 3. Tag \\
\hline \multicolumn{4}{|c|}{ Apoplektische Insulte ( $n=49)$} \\
\hline$\frac{\mathrm{CK}-\mathrm{MB}}{\mathrm{CK}_{\text {gesamt }}}$ & $\begin{array}{l}0.034 \\
(0-1,03)^{*}\end{array}$ & $\begin{array}{l}0,036 \\
(0-1,26)^{*}\end{array}$ & $\begin{array}{l}0,022 \\
(0-1,05)^{*}\end{array}$ \\
\hline \multicolumn{4}{|c|}{ Schädel-Hürn-Träumen ( $n=39)$} \\
\hline$\frac{\mathrm{CK}-\mathrm{MB}^{\circ}}{\mathrm{CK}_{\text {gesamt }}}$ & $\begin{array}{l}0,172 \\
(0-0,644)\end{array}$ & $\begin{array}{l}0,031 \\
(0-0,18.2)\end{array}$ & $\begin{array}{l}0,030 \\
(0-0,227)\end{array}$ \\
\hline
\end{tabular}

*Extremwerte bei der Mittelwertbildung nicht berücksichtigt 


\section{Literaturverzeichnis}

1. Szász, G. \& Busch, E. W. (1971), Z. Klin. Chem. Klin. Biochem. 9, 281.

2. Szász, G., Busch, E. W. \& Farohs, H. B. (1971), Dtsch. Med. Wochenschr. 96, 980-989.

3. Anido, V., Conn, R. B., Megoli, H. F. \& Anido, G. (1974), Amer. J. Clin. Pathol. 61, 599-605.

4. Roe, C. R., Limbird, L. E., Wagner, G. S. \& Nerenberg, S. T. (1972), J. Lab. Clin. Med. 80, 577-590.

5. Mercer, D. W. \& Varat, M. A. (1975), Clin. Chem. 21, 10881092.

6. Würzburg, U., Hennrich, N., Lang, H., Prellwitz, W., Neumeier, D. \& Knedel, M. (1976), Klin. Wochenschr. 54, $357-360$.

7. Neumeier, D., Prellwitz, W., Wirzburg, U., Brundobler, M., Olbermann, M., Just, H. I., Knedel, M. \& Lang, H. (1976), Clin. Chim. Acta 73, 445-451.

8. Prellwitz, W., Kapp, S., Neumeier, D., Knedel, M., Lang, H. \& Heuwinkel, D. (1978), Klin. Wochenschr. 56, 559-565.

9. Dubo, H., Park, D. C. \& Pennington, I. T. (1967), Lancet II, 743-748.

10. Jockers-Wretou, Evangelia \& Pfleiderer, G. (1975), Clin. Chim. Acta 58, 223-232.

11. Sobe, D., Scheidt, F., Haux, P. \& Kattermann, R. (1977), J. Clin. Chem. Clin. Biochem. 15, 188.

12. Würzburg, U. \& Prellwitz, W. (1977), J. Clin. Chem. Clin. Biochem. 15, 198.

13. Klapdor, R., Harm, K. \& Kugler, G. (1977), Dtsch. Med. Wochenschr. 102, 1309-1314.

14. Weidemann, G. \& Schmid, R. D. (1978), J. Clin. Chem. Clin. Biochem. 16, 253-254.

15. Knob, Margarethe \& Rosenmund, H. (1978), Schweiz. Med. Wochenschr. 108, 788-791.
16. Empfehlungen der Deutschen Gesellschaft für Klinische Chemie: Standardisierung von Methoden zur Bestimmung von Enzymaktivitäten in biologischen Flüssigkeiten (1972), Z. Klin. Chem. Klin. Biochem. 10, 182-192.

17. Neumeier, D., Prellwitz, W., Sandel, P. \& Knedel, M. (1978), Klin. Wochenschr. 56, 449-456.

18. Neumeier, D., Knedel, M., Würzburg, U., Hennrich, N. \& Lang, H. (1975) Klin. Wochenschr. 53, 329-333.

19. Chemnitz, G., Schmidt, E., Koller, P. U. \& Busch, E. W. (1979), Dtsch. Med. Wochenschr. 104, 257-260.

20. Trautschold, I. (1975). In: Anwendung immunologischer Methoden (Lang, H., Rick, W. \& Roka, L., eds.) 240-241, Springer-Verlag, Berlin-Heidelberg-New York.

21. Neumeier, D. \& Knedel, M. (1976), CK-Isoenzym-Symposium, Wien 16. Oktober.

22. Flenker, I., Ricken, D. \& Sabin, G. (1977), Diagnostik 10, 752-755.

23. Schmidt, E. W. \& Bender, W. (1979), J. Clin. Chem. Clin. Biochem. 17, 9-13.

24. Schmidt, E. W., Wellstein, A. \& Moll, A. (1977), Med. Klin. 72, 1368-1371.

25. Lang, H., Würzburg, U., Neumeier, D., Knedel, M., Prellwitz, W., Kattermann, R., Schlebusch, H. \& Schürmann, J. (1978), Klin. Wochenschr. 56, 641-646.

26. Gerhardt, W. \& Waldenström, J. (1979), Clin. Chem. 25, $1274-1280$.

27. Frotscher, U., Dominik, B., Richter, R., Zschaege, B., Messerschmidt, W., Schulte-Lippern, M., Schmidtmann, W. \& Willbrandt, R. (1973), Klin. Wochenschr. 51, 801-805.

28. Lorentz, K. (1979), J. Clin. Chem. Clin. Biochem. 17, 178-179.

29. Hackl, J. M., Puschendorf, B., Dienstl, F., Dworzak, E. \& Haid, B. (1978), Infusionstherapie 5, 204-207.

Dr. P. Haux, Prof. Dr. R. Kattermann Klinisch-Chemisches Institut Klinikum Mannheim der Univ. Heidelberg Postfach 23

D-6800 Mannheim 1 
